\title{
On the Optimization of Power Assignment to Support Multicast Applications in HAP-based Systems
}

\author{
Giuseppe Araniti, Antonio Iera, Antonella Molinaro \\ ARTS Laboratory - DIMET Department \\ Università Mediterranea di Reggio Calabria \\ Reggio Calabria, Italy \\ \{araniti, antonio.iera, antonella.molinaro\}@unirc.it
}

\author{
Alessandro Raschellà, Anna Umbert \\ Signal Theory and Communication Dept. \\ Universitat Politecnica de Catalunya \\ Barcelona, Spain \\ \{alessandror, annau $\} @$ tsc.upc.edu
}

\begin{abstract}
The goal of this research work is to investigate how efficient High Altitude Platforms (HAPs) can be in supporting Multimedia Broadcast/Multicast Service (MBMS) in scenarios in which the terrestrial coverage is not available. Specifically, we propose to implement an effective Radio Resources Management (RRM) policy into the HAP Radio Network Controller (H-RNC), whose main aim is to increase the overall system capacity. The proposed technique achieves its goal by dynamically selecting the most efficient multicast transport channel in terms of power consumption, chosen amongst Dedicated Channel (DCH), Forward Access Channel (FACH), and High Speed Downlink Shared Channel (HS-DSCH). Advantages deriving from the joint use of channels belonging to different categories are exploited. Results achieved when using the proposed RRM are quite manifest and witnesses to the necessity of providing such a feature when deploying integrated HAP/Terrestrial platforms supporting MBMS services.
\end{abstract}

Keywords-Multicast, MBMS, High Altitude Platforms, Radio Resource Management, HSDPA.

\section{INTRODUCTION}

Third Generation (3G) cellular wireless networks, such as Universal Mobile Telecommunication System (UMTS), have been designed upfront to supply at acceptable costs a wide range of "value-added" services, such as videoconference, multimedia streaming, broadband transmission, and multimedia file downloading [1]. The introduction of novel applications has raised the need of Point-to-Multipoint (PtM) (i.e. among one sender and several receivers) communications. The use of either multicast or broadcast technologies is an efficient method to support PtM services. For this reason, the third generation partnership project (3GPP) introduced a new system, called Multimedia Broadcast/Multicast Service (MBMS), into the UMTS standard finalized to the provision of group oriented services. Nonetheless, a terrestrial MBMS segment could lead to some inefficiency in environments with high exacting communication requirements. This is the case of the so called disadvantaged areas, such as rural areas or areas involved in unpredictable catastrophic accidents. Hence, the integration of space segments into the MBMS architecture is gaining ground and is attracting a special attention from the scientific and industrial communities.
High Altitude Platforms (HAPs) are space segments, usually located at an altitude of $17-22 \mathrm{~km}$, particularly suited to support MBMS services and applications in disadvantages areas not easily covered by using traditional terrestrial networks. Some of the HAP's advantages are: relatively low platform upgrading cost, broadband capability, large area coverage, flexibility to respond to traffic demands, low propagation delay and incremental deployment [2]. HAPs could be utilized in two different ways: $(i)$ in the standalone way as base stations in the sky; or (ii) in the integrated way as an overlapping coverage for available terrestrial systems.

In this research work we consider the standalone case only and focus on how HAPs can efficiently support MBMS services in rural/emergency scenarios. In particular, we aim at implementing an efficient Radio Resources Management (RRM) policy that allows reducing as much as possible the HAP power consumption assigned to a multicast group. The obvious power constraints in delivering multicast traffic pushes through a wise selection of the most efficient transport channel. MBMS services might be supported over the radio interface either by Forward Access Channels $(\mathrm{FACH})$, or Dedicated Channels (DCH), defined by UMTS Release 99, or High Speed Downlink Shared Channels (HS-DSCH), subsequently introduced by High Speed Downlink Packet Access (HSDPA) Release 5.

Several research works have been conducted, targeted to the reduction of the consumed power for MBMS data delivery, which, however, takes into account the UMTS terrestrial network only. For instance, in [3-5] the authors carry out studies for the selection of the most efficient transport channel depending on several issues, such as application bit rate and user positions. Instead, in [6] the authors propose to set the FACH transmission power depending on the location of the farthest user belonging to the multicast group; while in [7] a mixed usage of multiple DCH and FACH channels is suggested with the aim of saving transmission power. On the other hand, studies are present in the literature with the goal to define the most efficient transport channel when considering the integration of HAPs into the MBMS architecture, amongst Dedicated, Shared, and Common channels but without any mixed usage of them $[8,9]$. 
By starting from the referred studies, the present paper has the following purpose: when any new user joins a multicast group, the RRM policy that we propose has to determine the most efficient transport channel to assign to her/him, depending on her/his position, the application bit rate, and the number of multicast users already belonging to the group. Intended contributions, compared to the state of the art, can be summarized as follows: (i) possibility of selecting a mixed usage of channels as the most efficient transport channels for MBMS transmissions from a space segment in rural/emergency scenarios; (ii) exploitation of the HS-DSCH channel and demonstration that its use can lead to an improvement of the MBMS performance in terms of wasted power.

The paper is organized as follows. Section II provides an overview of the integration of HAPs into the MBMS architecture. Section III introduces the transport channels utilized for multicast transmissions in the HAP system segment. A novel RRM policy is proposed in Section IV, while the main results of a comprehensive simulation campaign, aiming at assessing the achievable performance, are the focus of Section V. Conclusive remarks are given in Section VI.

\section{MBMS/HAP ARCHITECTURE}

In the considered scenario we take into account HAPs that carry a UMTS payload at an altitude of $22 \mathrm{~km}$ above the service area. Moreover, we omit the effect of the Earth's curvature and suppose an array of elliptical beam antennas on board the HAPs illuminating the service area with circular cells of radius $r$ mapped onto hexagonal grids, according to [10-12]. The exact shape of such cells is given by the antenna radiation pattern. Aerial antennas are used to cover several terrestrial cells, creating an aerial macrocell, as shown in Fig. 1 ; we consider only the first tier (consisting of 6 cells) because we have checked that the interference derived from the second tier does not affect the results of our simulations.

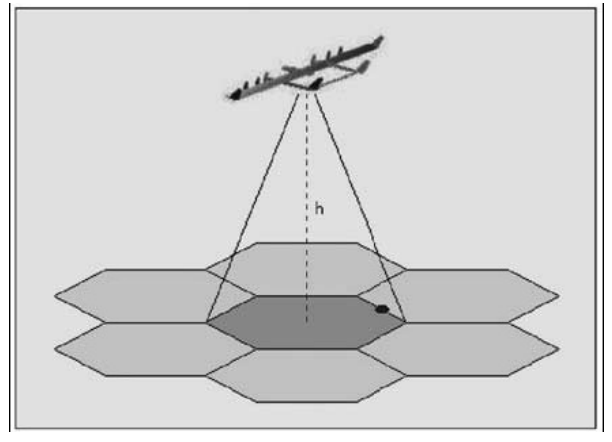

Figure 1. Aerial Macrocell

A possible way to include HAPs into the MBMS architecture to provide multicast/broadcast services is shown in Fig. 2. Such an augmentation of the terrestrial system implies just the addition of an UMTS-HAP Control System (U-HCS) to the MBMS architecture, with functionality of HAP Radio Network Controller (H-RNC).

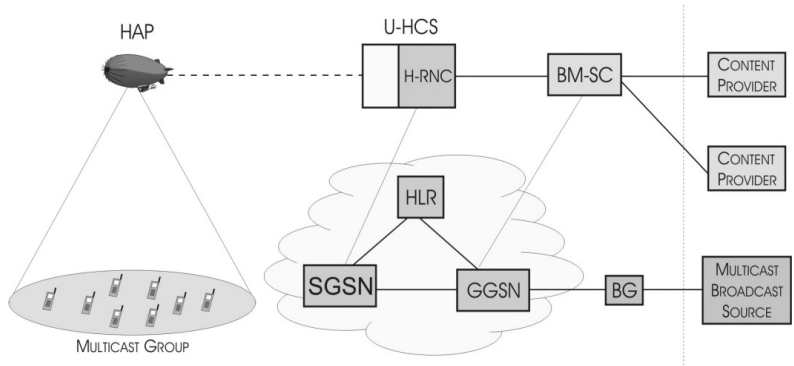

Figure 2. UMTS/HAPs Integrated Systems for Multicast Services Delivery

In particular, we remark the presence of the following elements: SGSN (Serving GPRS Support Node) that performs user individual service control functions and gathers together all individual users of the same MBMS service into a $M B M S$ group; GGSN (Gateway GPRS Support Node) that completes the MBMS GTP (GPRS tunnelling protocol) tunnels from the SGSN and links these tunnels via IP multicast with the MBMS data source; BM-SC (Broadcast-Multicast Service Centre) that is the novel component of the architecture introduced to provide MBMS data.

The proposed MBMS/HAP architecture is apparently very similar to a MBMS/UMTS terrestrial network; although, several differences (as reported in Table I) between terrestrial and space segments shall be taken into account in the determination of the most efficient transport channel $[13,14]$.

The most evident difference is represented by the propagation environment. Indeed, HAPs enjoy more favorable Path Loss characteristics compared to wireless terrestrial links; in fact in the Free Space case affected by Rooftop Scattering and Multiple Screen Diffraction the received power decays as a function of the transmitter-receiver distance raised roughly to a power of 2 . While, in wireless terrestrial systems, where the Okumura Hata model must be taken into account [1], the received power decays as a function of the transmitterreceiver distance raised to a power of 4 .

TABLE I. DIFFERENCES BETWEEN TERRESTRIAL AND SPACE SEGMENTS

\begin{tabular}{|c|c|c|}
\hline Features & Terrestrial Wireless & HAP \\
\hline $\begin{array}{c}\text { Breadth of } \\
\text { geographical } \\
\text { coverage }\end{array}$ & $\begin{array}{c}\text { A few kilometres per } \\
\text { base station }\end{array}$ & $\begin{array}{c}\text { Hundreds of } \\
\text { kilometres per } \\
\text { platform }\end{array}$ \\
\hline Cell diameter & $0.1-1 \mathrm{~km}$ & $1-10 \mathrm{~km}$ \\
\hline $\begin{array}{c}\text { Shadowing from } \\
\text { terrain }\end{array}$ & $\begin{array}{c}\text { Causes gaps in } \\
\text { coverage }\end{array}$ & $\begin{array}{c}\text { Only at low elevation } \\
\text { angles }\end{array}$ \\
\hline Path Loss & Okumura Hata & $\begin{array}{c}\text { Free Space affected } \\
\text { by Rooftop Scattering } \\
\text { and Multiple Screen } \\
\text { Diffraction }\end{array}$ \\
\hline
\end{tabular}




\section{TRANSPORT CHANNELS SUPPORTED BY MBMS/HAP}

As already mentioned, the MBMS data can be delivered to users by utilizing any of three different transport channels provided by UMTS: DCH, FACH, and HS-DSCH. Hence, we briefly recall the modality to assign the transmission power to such channels. The transmission power assigned to the DCHs can vary depending on: $(i)$ the multicast users number; (ii) the users position in respect to the centre of the area covered by the HAP; (iii) the bit rate of the application.

In (1) it is shown how to define the transmission power assigned to a DCH serving the "i_th" user in a given cell (named own cell in the following) [15]:

$$
P_{D C H, i}=(C / I)_{i} \frac{X+Y+Z+N_{d}}{G_{i}^{o w n}\left(\theta_{i}, d_{i}\right)}(1)
$$

The terms X, Y e Z are explicated respectively in (2), (3) and (4).

$$
\begin{gathered}
X=\sum_{j=1}^{N_{\text {other }} \text { cell }} P_{j}^{\text {other }} G_{j}^{\text {other }}\left(\theta_{i}, d_{i}\right) \\
Y=p \sum_{k=1}^{N_{\text {user }}} P_{k}^{\text {own }} G_{k}^{\text {own }}\left(\theta_{i}, d_{i}\right) \\
Z=P^{\text {com }} G_{i}^{\text {own }}\left(\theta_{i}, d_{i}\right)
\end{gathered}
$$

the terms reported in such equations represent: $(i)$ the interference due to the transmitted power $\left(P_{j}^{\text {other }}\right)$ from the j_th adjacent cell belonging to the first tier (the total number of interfering cells is $\mathrm{N}_{\text {other_cell }}$ ); (ii) the interference due to the transmission power $\left(P_{k}^{\text {own }}\right)$ from the k_th user belonging to the multicast group that receive data within the own cell (the total number of own cell users is $\mathrm{N}_{\mathrm{user}}$ ); (iii) the interference due to the power used for transmission over the downlink common control channels $\left(P^{\text {com }}\right)$; (iv) the Additive Gaussian White Noise (AGWN) $\left(\mathrm{N}_{\mathrm{d}}\right)$. Furthermore, $p$ is the orthogonality factor which can be zero in case of perfect orthogonality, $G_{i}^{\text {own }}\left(\theta_{i}, d_{i}\right)$ is the link gain related to the i_th user with respect to its own cell, $G_{j}^{\text {other }}\left(\theta_{i}, d_{i}\right)$ is the link gain with respect to the neighboring cells while $G_{k}^{\text {own }}\left(\theta_{i}, d_{i}\right)$ is the one in the own cell; both are still related to the i_th user.

In general, $\mathrm{G}(\theta, \mathrm{d})$ and $\mathrm{C} / \mathrm{I}$ (the Carrier-to-Interference ratio) are defined respectively by (5) and (6).

$$
\begin{aligned}
& G(\theta, d)_{d B}=g(\theta)_{d B}-L_{p}(d)_{d B}(5) \\
& (C / I)_{d B}=\left(E_{b} / N_{0}\right)_{d B}-\left(P_{g}\right)_{d B}(6)
\end{aligned}
$$

$\theta$ is the angle representing the boresight direction, $g(\theta)$ is the Antenna Gain calculated in the boresight direction, $L_{p}(d)$ is the attenuation value caused by the Path Loss for the user at a distance equal to $d$ from the centre of the HAP coverage area. $E_{b} / N_{o}$ is the Energy per Bit-to-Spectral Noise Density while $P_{g}$ is the Processing Gain.

The FACH is a PtM channel with a power level high enough to guarantee an acceptable service in the whole coverage area [16]; it transmits at a fixed power level since fast power control is not supported in this channel. Both bit rate of the MBMS services and the cell coverage area affect the power allocated to FACHs. High data rate MBMS services might not be deliverable by $\mathrm{FACH}$, since excessive downlink transmission power would be required. High bit rates can only be offered to users located very close to the centre of the area covered by the HAP.

HS-DSCH is the shared transport channel that HSDPA exploits to deliver user data in the downlink direction. The main purpose of HSDPA technology is the increase of packet data throughput and rate. This goal is pursued thanks to link adaptation and fast physical layer retransmission. Furthermore, in HSDPA the Adaptive Modulation and Coding $(A M C)$ replaces the variable Spreading Factor $(S F)$ and the fast power control procedure. The $A M C$ allows changing the kind of modulation and coding depending on the radio channel conditions; such information is represented by the Channel Quality Information (CQI). In fact, the transport block size, the number of used physical channels, and the utilized modulation technique are valuated from the $C Q I$ value [17].

In a wireless network environment, the selected $E_{b} / N_{0}$ corresponds to a particular Block Error Rate (BLER) for the considered application bit rate. Notwithstanding, the $E_{b} / N_{0}$ metric is not an appropriate measure for HSDPA, because the bit rate over the HS-DSCH can vary every Transmission Time Interval (TTI). Therefore, the $E_{b} / N_{0}$ is replaced by the Signal to Interference Noise Ratio (SINR) representing a more appropriate measurement metric when considering the HSDSCH. Equation (7) defines such a parameter:

$$
S I N R=S F_{16} \frac{G(\theta, d) P_{H S-D S C H}}{p P_{\text {own }}+P_{\text {other }}+N_{d}}
$$

where $P_{H S-D S C H}$ is the transmission power to the HS$\mathrm{DSCH}, P_{\text {own }}$ is the own cell interference, $P_{\text {other }}$ is the interference caused by the cells belonging to the first tier, $N_{d}$ is the AGWN, $p$ is the orthogonality factor (that can be zero in the case of perfect orthogonality) and $S F_{16}$ is the SF that is equal to 16 [17]. Moreover, it is worth to mention the geometry factor $G F$, evaluated according to (8):

$$
G F=\frac{P_{o w n}}{P_{\text {other }}+N_{d}}
$$

Such a parameter represents the distance from the base station; in particular, a higher G-factor is expected when a user 
is closer to the center of the cell, where the own cell interference is higher than the interference from the neighboring cells. By rearranging (7) and considering (8), we can define the HS-DSCH SINR by the following Equation:

$$
S I N R=S F_{16} \frac{G(\theta, d) P_{H S-D S C H}}{P_{o w n}} \frac{1}{p+G F^{-1}}
$$

SINR is an important parameter for our research work; in fact, as explained later, it influences the CQI that can be selected, which in turn affects the maximum number of users that the HS-DSCH can serve.

\section{RRM POLICY}

The H-RNC module is responsible for the allocation of transport channels in the HAP segment. In case of resource allocation to multicast users, it decides whether several DCHs, or a HS-DSCH, or a FACH channel shall be activated over the HAP air interface. Indeed, a key issue to improve the overall system performance is the implementation of an effective RRM algorithm into the H-RNC. The basic behavior of the proposed resource management policy is quite obvious. When a new multicast user enters the cell served by the HAP the system checks her/his position, the application bit rate, and the number of multicast users already active in the same area. According to the information retrieved, the system assigns to the new user the most suitable transport channel for the MBMS session.

In our research work we propose a mixed usage of channels, and we suppose that the FACH is already active to provide a multicast service to the users scattered within only a part of the cell coverage area. A user located in the remaining area of the cell could be served by using either DCHs or HS$\mathrm{DSCH}$, or even by enlarging the area covered by FACH. Therefore, the key research point is related to the choice of the transport channel to assign to the new user, taken among the three following options:

\section{Assignment of a DCH;}

\section{Assignment of the HS-DSCH;}

\section{Extension of the FACH.}

The behavior of the proposed RRM policy consists of the operational phases illustrated in Figure 3: (i) Data Retrieval Phase; (ii) Data Computation Phase; (iii) Channel Selection Phase.

In particular, when a new multicast user joins a multicast group, the following parameters are retrieved in the Data Retrieval Phase: the power assigned to the HS-DSCH channel $\left(P_{H S-D S C H}\right)$, the application bit rate, the multicast user position (supposed to be outside the part covered by the FACH), and the number of multicast users already active in the same area (then outside the part already covered by the $\mathrm{FACH}$ ) named Active Users Number (AUN).
Such parameters are the input of the Data Computation Phase. In particular, the following values are computed: the Maximum Users Number (MUN) that can be served by the HS-DSCH, the power used by the already active $\mathrm{FACH}$ $\left(P_{F A C H}\right)$, an amount of transmission power used to keep alive a number of DCHs equal to $A U N$ plus a further amount utilized by the already active FACH $\left(P_{D C H_{-} F A C H}\right)$, the transmission power value enabling to extend the FACH coverage $\left(P_{E F A C H}\right)$ and get the position of the new user.

Such information allows the RRM policy selecting the most efficient transport channel in terms of consumed power during the subsequent phase, named Channel Selection Phase. Since we suppose that the broadcast channel is already active to cover a part of the cell, such a phase foresees a first step to select the most efficient transport channel between DCH and HS-DSCH for the users located in the outer part. While in the subsequent step, it is decided whether such a selected channel or, instead, the extension of the FACH coverage is the most appropriate solution in terms of consumed power.

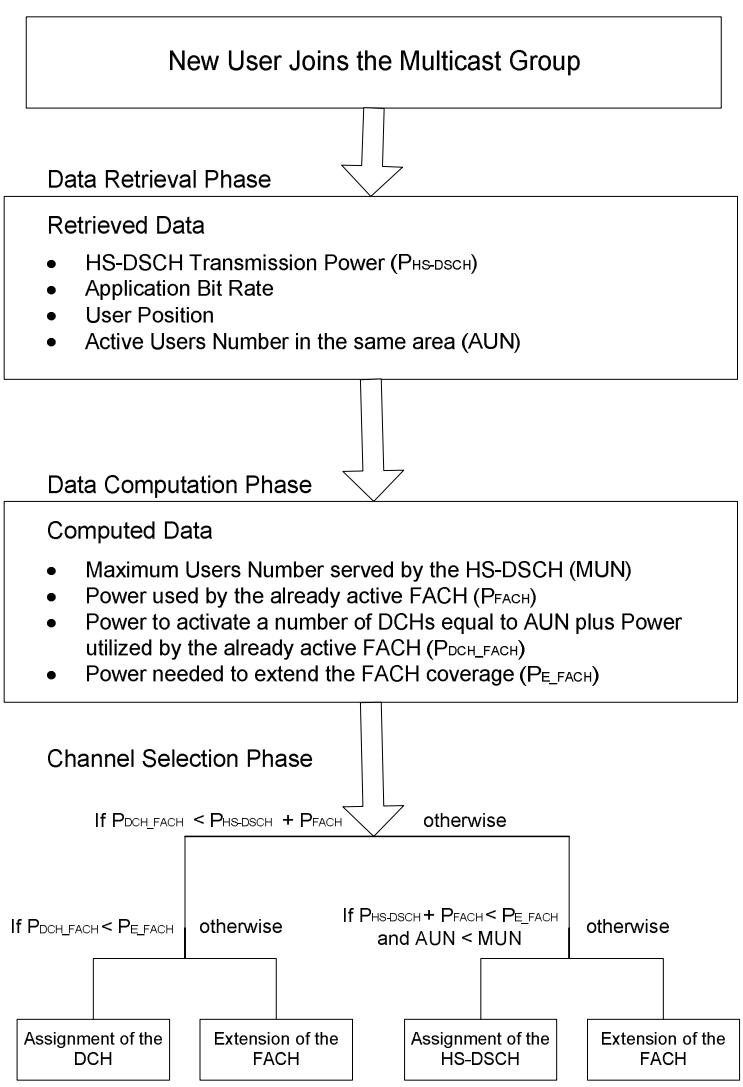

Figure 3. RRM algorithm mechanism

In general, the selection of the transport channel depends on the minimization of the consumed transmission power. Notwithstanding, it may happen that $A U N$ becomes greater than $M U N$ (this means that the number of active multicast 
users overcomes the maximum number of users that can be served by the HS-DSCH, remember that it is a shared channel); in this case, the $\mathrm{FACH}$ coverage is extended regardless of the consumed power to be able to serve all the new users joining the multicast group (remember that the FACH is a broadcast channel).

The study illustrated in the next section includes an exhaustive evaluation and assessment campaign, aimed at establishing the most suitable resource allocation policy that shall be adopted in any situation and at demonstrating how the designed policy leads to a manifest power saving. In our study, we suppose the FACH channel to be already active to initially cover $50 \%$ of the cell coverage, according to the output of early studies reported in [9]. This assumption does not reduce the generality of the overall results, which do not change substantially when a different initial FACH coverage level is foreseen in the cell.

\section{EVALUATION CAMPAIGN}

Table II summarizes the main assumptions of our research $[14,18,19]$. The main purpose of this research work is to support 64 and $128 \mathrm{kbps}$ services based on a mixed usage of multiple DCHs, HS-DSCH, and FACH channels to serve multicast users. Since we suppose that the FACH channel is initially active to cover $50 \%$ of the cell coverage size, in Table III we reported the threshold number of multicast users that triggers the switching of a given multicast service from DCHs to the broadcast channel within such a cell coverage, considering 64 or $128 \mathrm{kbps}$ application bit rate in according to [9]. The reader can notice that in these cases the HS-DSCH is not used because DCHs and FACH are more appropriate in terms of transmission power.

TABLE II. SimULATION CAMPAIGN ASSUMPTIONS

\begin{tabular}{|c|c|}
\hline Parameter & Value \\
\hline HAP High & $22 \mathrm{Km}$ \\
\hline Cell Radius & $2.6 \mathrm{Km}$ \\
\hline Cell Layout & Hexagonal grid \\
\hline Number of Neighboring Cells & $40 \mathrm{~W}$ \\
\hline Maximum HAP Tx Power & $10 \mathrm{~W}$ \\
\hline Other BS Tx Power & $2 \mathrm{~W}$ \\
\hline Common Channel Power & $\begin{array}{c}\text { Free Space affected by Rooftop } \\
\text { Scattering and Multiple Screen } \\
\text { Diffraction }\end{array}$ \\
\hline Path Loss & Vehicular A (3Km/h) \\
\hline Multipath Channel & 0.5 \\
\hline Orthogonality Factor & $10 \%$ \\
\hline BLER Target & $32.3 \mathrm{dBi}$ \\
\hline Gmax & $-100 \mathrm{dBm}$ \\
\hline Thermal Noise &
\end{tabular}

TABLE III. SWITCHING THRESHOLDS WITHIN 50\% CELL COVERAGE

\begin{tabular}{|c|c|c|}
\hline Bit Rate (kbps) & Number of Users $\left(\mathbf{N}_{\mathbf{u}}\right)$ & Utilized Channel \\
\hline \multirow{2}{*}{64} & $\mathbf{N}_{\mathbf{u}} \leq 33$ & DCHs \\
\cline { 2 - 3 } & $\mathbf{N}_{\mathbf{u}}>33$ & FACH \\
\hline \multirow{2}{*}{128} & $\mathbf{N}_{\mathbf{u}} \leq 22$ & DCHs \\
\cline { 2 - 3 } & $\mathbf{N}_{\mathbf{u}}>22$ & FACH \\
\hline
\end{tabular}

Hence, as a next step, we assume the number of users greater than 33 and 22 within $50 \%$ cell coverage, for 64 and $128 \mathrm{kbps}$ services respectively. This means that in this portion of coverage area a FACH supports multicast data delivery.

Under these assumptions, next objective is the selection, according to the functional diagram in Fig. 3, of the most efficient transport channel when a new user joins the multicast group outside the $50 \%$ of the cell coverage. During the Data Retrieval Phase the H-RNC retrieves the $P_{H S-D S C H}$, the application bit rate (that can be either 64 or $128 \mathrm{kbps}$ ), the user position (remember that in the simulation campaign it is assumed to be outside the initial FACH coverage), and the $A U N$ value. Subsequently, during the Data Computation Phase, the H-RNC computes the required power values from the parameter values previously obtained.

Fig. 4 and 5 depict the HAP overall transmission power as a function of the multicast users scattered across the cell, for 64 and $128 \mathrm{kbps}$ application bit rates, respectively. In particular, both figures depicts the following power values: $(i)$ the power needed by the FACH to cover $50 \%$ cell coverage; (ii) the power required to offer services by using the $\mathrm{FACH}$ to cover $95 \%$ cell coverage; (iii) the power assigned to the DCHs depending on the number of users scattered within $95 \%$ coverage and considering also the interference from the FACH active to cover $50 \%$ cell coverage. From such figures we can obtain $P_{F A C H}, P_{D C H F F C H}$, and $P_{E F A C H}$ when Application Bit Rate, User Position, and $A U N$ are known.

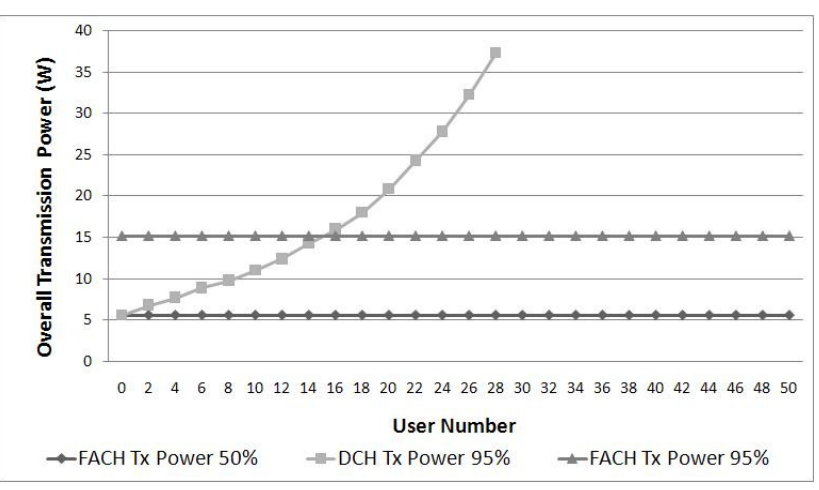

Figure 4. Mixed Usage of Channels for $64 \mathrm{kbps}$

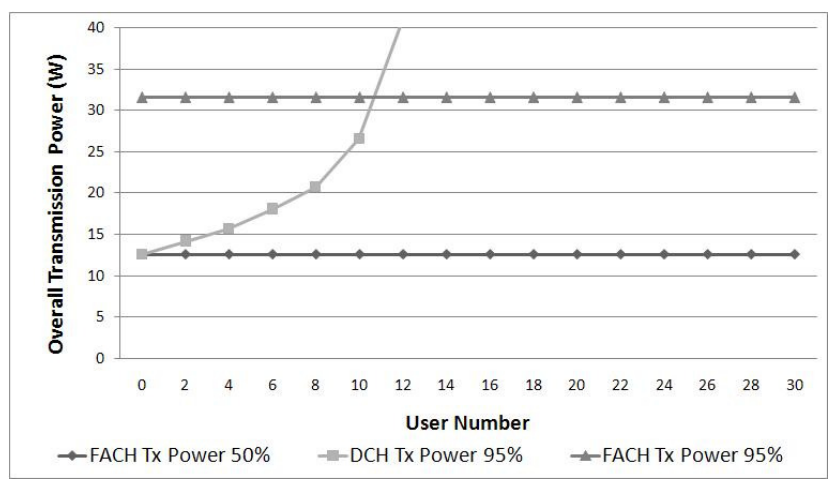

Figure 5. Mixed Usage of Channels for $128 \mathrm{kbps}$ 
From (9) it emerges that the SINR depends both on the assigned HS-DSCH transmission power and on the area served by the channel; SINRs obtained by fixing such parameters limit the MUN value. Fig. 6 illustrates the SINRs to be guaranteed, when varying the assigned power and fixing the $95 \%$ cell coverage; moreover, since we are supposing that the FACH channel is active to cover $50 \%$ cell coverage, its interference is also taken into account. As expected from (9), the guaranteed SINR improves when the HS-DSCH assigned power increases.

Subsequently, we highlight how obtained SINRs limit the $M U N$ value. In particular, in Table IV we list the following parameter values: $(i)$ the CQIs obtained depending on the guaranteed SINRs, given a target BLER of 10\%; (ii) a mapping between the CQI values and the $M U N$ value. Such a number is obtained by dividing the maximum Physical Bit Rate by the service bit rate [17].

For instance, the CQI equal to 5, characterized by a QPSK modulation and by a use of one physical channel, gives a maximum Physical Bit Rate equal to $480 \mathrm{kbps}$ that allows serving 7 User Equipments (UEs) for $64 \mathrm{kbps}$ applications and 3 UEs for $128 \mathrm{kbps}$ services. Hence, we can achieve the $M U N$ value considering $P_{H S-D S C H}$ and User Position derived from the Data Retrieval Phase.

The HS-DSCH transmission power value can be set according to two different modalities. The H-RNC can explicitly allocate an amount of transmission power to the HSDSCH and update such a value any time later. Otherwise, the $\mathrm{H}$-RNC allows using any unused power in the cell (i.e. the residual power after serving other channels) for the HSDSCH. In our research work we assume the first option.

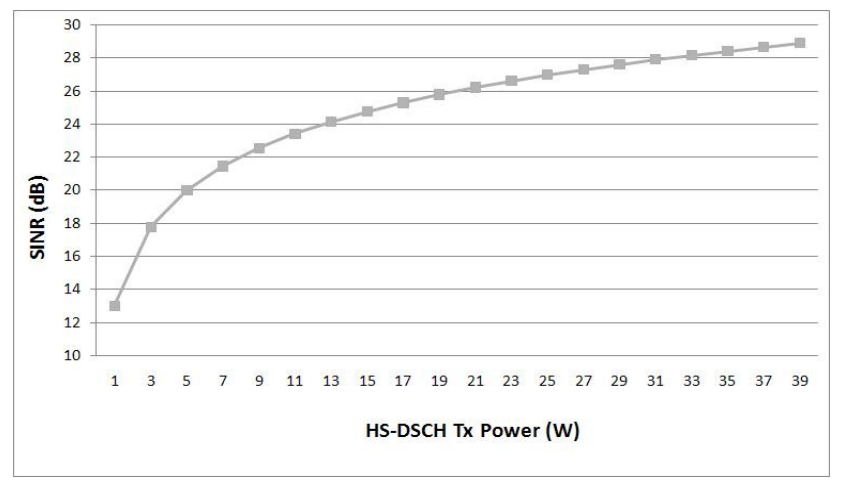

Figure 6. SINR vs HS-DSCH Transmission Power

The computed power values are used as input to the next phase, namely Channel Selection Phase, to allow the H-RNC assigning a new user the most efficient transport channel.

To highlight the performance gain obtained by the proposed RRM algorithm, we focus our attention on a particular case. A new user joins the multicast group and, during the Data Retrieval Phase, the H-RNC becomes aware of the following conditions: $P_{H S-D S C H}$ equal to $9 \mathrm{~W} ; 64 \mathrm{kbps}$ application bit rate; new user located outer $50 \%$ of the cell coverage; $A U N$ equal to 20. Next, during the Data Computation Phase, the system computes the following values: $M U N$ equal to 22 (see Fig. 6 and Table IV); $P_{F A C H}$ equal to $5.6 \mathrm{~W}$ (see Fig. 4); $P_{D C H_{-} F A C H}$ equal to $21 \mathrm{~W}(5.6 \mathrm{~W}$ for the already active $\mathrm{FACH}$ to serve $50 \%$ of the cell coverage plus $15.4 \mathrm{~W}$ to serve $20 A U N$ located within $95 \%$, according to Fig. 4); $P_{E_{-} F A C H}$ equal to $15.2 \mathrm{~W}$ (see Fig. 4). At this point, the system is able to select the most efficient transport channel in terms of consumed power for the new user: since $P_{H S-D S C H}$ plus $P_{F A C H}$ is lower than both $P_{D C H_{-} F A C H}$ and $P_{E_{-} F A C H}$ and $A U N$ is lower than $M U N$, then the wiser channel assignment to the new user is undoubtedly the HS-DSCH.

TABLE IV. HS-DSCH PARAMETERS

\begin{tabular}{|c|c|c|c|c|c|c|}
\hline $\begin{array}{c}\text { SINR } \\
(\text { dB })\end{array}$ & CQI & Mod & $\begin{array}{c}\# \\
\text { Ph. } \\
\text { Ch. }\end{array}$ & $\begin{array}{c}\text { Physical } \\
\text { Bit Rate } \\
\text { (kpbs) }\end{array}$ & $\begin{array}{l}\text { MUN } \\
\text { 64kbps }\end{array}$ & $\begin{array}{c}\text { MUN } \\
\text { 128kbps }\end{array}$ \\
\hline 21 & 5 & QPSK & 1 & 480 & 7 & 3 \\
\hline 22 & 8 & QPSK & 2 & 960 & 15 & 7 \\
\hline \multirow{2}{*}{23} & 10 & QPSK & 3 & 1440 & 22 & 11 \\
\hline & 11 & QPSK & 3 & 1440 & 22 & 11 \\
\hline \multirow{3}{*}{24} & 12 & QPSK & 3 & 1440 & 22 & 11 \\
\hline & 13 & QPSK & 4 & 1920 & 30 & 15 \\
\hline & 14 & QPSK & 4 & 1920 & 30 & 15 \\
\hline \multirow{8}{*}{25} & 15 & QPSK & 5 & 2400 & 37 & 18 \\
\hline & 16 & 16-QAM & 5 & 4800 & 75 & 37 \\
\hline & 17 & 16-QAM & 5 & 4800 & 75 & 37 \\
\hline & 18 & 16-QAM & 5 & 4800 & 75 & 37 \\
\hline & 19 & 16-QAM & 5 & 4800 & 75 & 37 \\
\hline & 20 & 16-QAM & 5 & 4800 & 75 & 37 \\
\hline & 21 & 16-QAM & 5 & 4800 & 75 & 37 \\
\hline & 22 & 16-QAM & 5 & 4800 & 75 & 37 \\
\hline 27 & 23 & 16-QAM & 7 & 6720 & 105 & 52 \\
\hline 28 & 24 & 16-QAM & 8 & 7680 & 120 & 60 \\
\hline 30 & 25 & 16-QAM & 10 & 9600 & 150 & 75 \\
\hline 32 & 26 & 16-QAM & 12 & 11520 & 180 & 90 \\
\hline \multirow{4}{*}{34} & 27 & 16-QAM & 15 & 14400 & 225 & 112 \\
\hline & 28 & 16-QAM & 15 & 14400 & 225 & 112 \\
\hline & 29 & 16-QAM & 15 & 14400 & 225 & 112 \\
\hline & 30 & 16-QAM & 15 & 14400 & 225 & 112 \\
\hline
\end{tabular}

How the overall system can benefit from the mixed usage of channels is illustrated in Fig. 7 and 8. In particular, in Fig. 7 is reported the power gain when the HS-DSCH is not considered. For instance, in case of $64 \mathrm{kbps}$ applications, when the first user joins the multicast group outside $50 \%$, then activating a DCH instead of extending the $\mathrm{FACH}$ coverage allows to save $9.2 \mathrm{~W}$ ( $23 \%$ of the overall transmission power).

The saved power decreases to $0.9 \mathrm{~W}(2.25 \%$ of the overall transmission power) when the last user joins the multicast group, just before the extension of the FACH coverage becomes the most suitable choice. In case of $128 \mathrm{kbps}$ application bit rate, maximum and minimum values of saved power are 18.3 and $10.8 \mathrm{~W}(45.75 \%$ and $27 \%$ of the overall transmission power) respectively. 


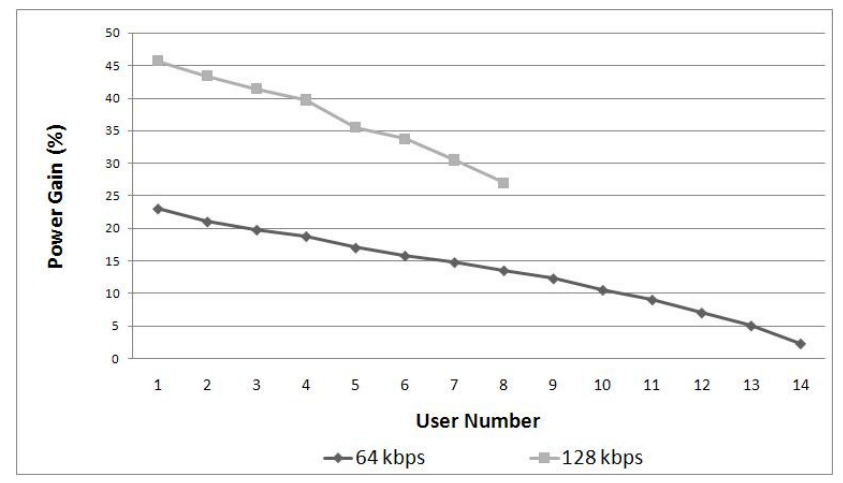

Figure 7. Power Gain depending on the Number of Users Scattered between $50 \%$ and $95 \%$ Cell Coverage

As a next step we want to assess that the introduction of the HS-DSCH can improve the performance of the mixed usage of channels adopted by the proposed RRM.

To better understand the effective role played by the HSDSCH in the proposed RRM policy, let us focus on the following sample situations. For $64 \mathrm{kbps}$ applications the envisaged RRM policy leads the system to select the FACH to cover 50\% and in addition the DCHs to cover up to 14 new multicast users outside 50\% cell coverage (according to the results illustrated in Fig. 4); while it drives the system to select the FACH to cover $50 \%$ plus the HS-DSCH when the number of multicast users is included between 15 and 22 (according to the results reported in Fig. 4 and Table IV); if such a number becomes greater than 22 then the RRM extends the coverage of the FACH up to $95 \%$. Therefore, if for instance the number of multicast users outside $50 \%$ was equal to 20 , then the introduction of the HS-DSCH in a mixed usage of channels lead to save $0.6 \mathrm{~W}$.

Things changes instead, for $128 \mathrm{kbps}$; in this case it emerges that the system selects the $\mathrm{FACH}$ to cover $50 \%$ plus the DCHs up to 8 new multicast users outside $50 \%$ cell coverage (according to the results illustrated in Fig. 5); it selects the FACH to cover $50 \%$ plus the HS-DSCH when the number of multicast users is included between 9 and 11 (according to the results reported in Fig. 4 and Table IV); while, if such a number becomes greater than 11 then the system extends the coverage of the FACH up to $95 \%$. In this case if for instance the number of multicast users outside $50 \%$ was equal to 10 , then the use of the HS-DSCH leads to save $10 \mathrm{~W}$.

Fig. 8 depicts how the overall system can benefit from the introduction of the HS-DSCH in a mixed usage of channels. From the figure we can draw the following conclusions: (i) when HS-DSCH is taken into account the FACH coverage is extended for a number of multicast users outside $50 \%$ higher than in the case when the shared channel is not considered (this means saving power); (ii) the introduction of the shared channel implies a power saving that could reach $25 \%$ of the overall transmission power $(10 \mathrm{~W})$; in particular such a maximum value is obtained when the number of multicast users is included between 9 and 11 , for $128 \mathrm{kbps}$ applications.

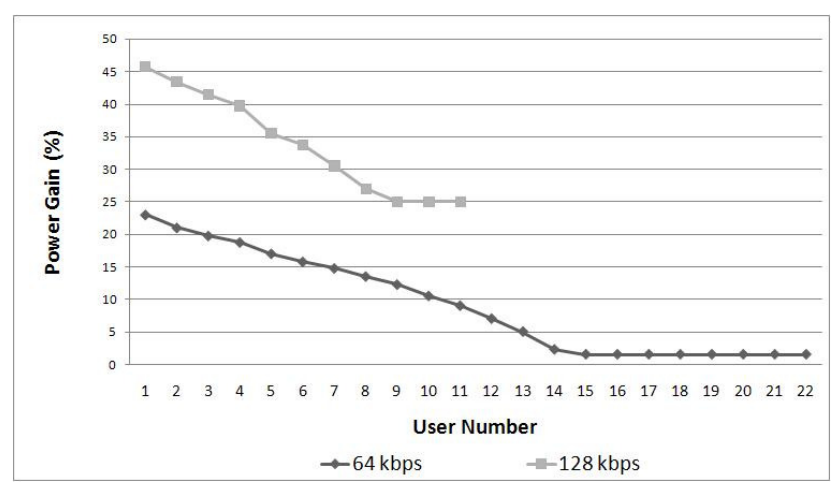

Figure 8. Power Gain (compared to the case in which no HS-DSCH is used) depending on the Number of Users Scattered between $50 \%$ and $95 \%$ Cell Coverage when considering also the HS-DSCH.

\section{CONCLUSION}

In providing MBMS services, the choice of the most efficient transport channel is a key aspect, since a wrong transport channel selection could adversely affect the overall capacity of the system. In this paper we defined a RRM policy aiming at identifying the most efficient transport channel in terms of consumed power among DCH, HS-DSCH, and $\mathrm{FACH}$, by considering the radio channel conditions, the cell coverage radius, and two MBMS application bit rates. In particular, we introduced a mixed usage of channels considering the main differences between terrestrial and space segment. Moreover, we took into account the HS-DSCH in such a mixed usage of channels highlighting how its presence can lead to an improvement of the MBMS performances in a HAP standalone scenario.

\section{ACKNOWLEDGMENT}

This work has been supported by Italian Research Program (PRIN 2007) Satellite-Assisted Localization and Communication system for Emergency services (SALICE). Web site: http://lenst.det.unifi.it/salice

\section{REFERENCES}

[1] H. Holma, A. Toskala, "WCDMA for UMTS - Radio Access for Third Generation Mobile Communications" John Wiley and Sons, 2004.

[2] K. Narenthiran, "S-UMTS access network for MBMS service delivery: the SATIN approach", International Journal of Satellite Communications and Networking, Jan.-Feb. 2004.

[3] A. Alexiou, C. Bouras, V. Kokkinos, E. Rekkas, "Power efficient radio bearer selection in MBMS multicast mode", MSWIM, 2007.

[4] IST-2001-35125 (OverDRiVE), Deliverable of the project (D08), "Spectrum Efficient Multicast and Asymmetric Services in UMTS".

[5] A. Raschellà, A. Umbert, G. Araniti, A. Iera, A. Molinaro, "SINR-based transport channel selection for MBMS application", VTC Spring, 2009.

[6] 3GPP TSG RAN WG1\#28 R1-02-1239 Espoo, Finland, 2002.

[7] A. Alexiou, C. Bouras, V. Kokkinos, "Power saving techniques in MBMS multicast mode", WMuNeP, 2007.

[8] A. Raschellà, G. Araniti, A. Iera, A. Molinaro "High altitude platforms: radio resource management policy for MBMS applications", PSATS, 2009. 
[9] A. Raschellà, G. Araniti, A. Iera, A. Molinaro "Radio resource management policy for multicast transmissions in high altitude platforms", ICSSC, 2009.

[10] Y.C. Foo, W.L. Lim, R. Tafazolli, L. Barclay, "Other-cell interference and reverse link capacity of high altitude platform station CDMA system", vol. 36, No. 22, Electronics Letters $26^{\text {Th }} 2000$.

[11] P. Pechac, "Outlook of the propagation of radiowaves", Technical Report, Czech Technical University in Prague, Faculty of Electrical Engineering.

[12] J. Holis, P. Pechac "Cell capacity of UMTS provided via HAP stations using elliptical and circular beam antennas in urban and suburban areas", In Proceedings in COST 297 WGI Meeting, 2006.

[13] S. Karapantazis, F.-N. Pavlidou Aristotle "Broadband communications via High Altitude Platforms (HAPs) - A survey", Communications Letters, IEEE, 2005.
[14] Iskandar, S. Shimamoto "On the performance of IMT-2000 communication link based on stratospheric platforms", Makara, Teknologi, vol. 10, No. 1, 2006, pp 1-10.

[15] B. Taha-Ahmed, M. Calvo-Ramòn, L. de Haro-Ariet "On the High Altitude Platfom (HAP) W-CDMA system capacity", Radio engineering, vol. 13, No.2, 2004.

[16] IST-2003-507607 (B-BONE). Deliverable D2.5. Final results with combined enhancements of the air interface.

[17] H. Holma, A. Toskala "HSDPA/HSUPA for UMTS - High Speed Radio Access for Mobile Communications", John Wiley and Sons, 2006.

[18] E. Falletti, M Mondin, F. Dovis, D. Grace "Integration of a HAP within a terrestrial UMTS network", Wireless Personal Communications, 2003.

[19] D. Grace, C. Spillard, J. Thornton, T.C. Tozer "Channel assignmen strategies for a high altitude platform spot-beam architecture", PIMRC, 2002. 